\title{
ARTICLE
}

\section{Long-range ordered vorticity patterns in living tissue induced by cell division}

\author{
Ninna S. Rossen ${ }^{1}$, Jens M. Tarp${ }^{1}$, Joachim Mathiesen ${ }^{1}$, Mogens H. Jensen ${ }^{1} \&$ Lene B. Oddershede ${ }^{1}$
}

In healthy blood vessels with a laminar blood flow, the endothelial cell division rate is low, only sufficient to replace apoptotic cells. The division rate significantly increases during embryonic development and under halted or turbulent flow. Cells in barrier tissue are connected and their motility is highly correlated. Here we investigate the long-range dynamics induced by cell division in an endothelial monolayer under non-flow conditions, mimicking the conditions during vessel formation or around blood clots. Cell divisions induce long-range, well-ordered vortex patterns extending several cell diameters away from the division site, in spite of the system's low Reynolds number. Our experimental results are reproduced by a hydrodynamic continuum model simulating division as a local pressure increase corresponding to a local tension decrease. Such long-range physical communication may be crucial for embryonic development and for healing tissue, for instance around blood clots.

\footnotetext{
${ }^{1}$ Niels Bohr Institute, University of Copenhagen, Blegdamsvej 17, DK-2100 Copenhagen, Denmark. Correspondence and requests for materials should be addressed to L.B.O. (email: oddershede@nbi.dk).
} 
E ndothelial cells line the blood vessels of the circulatory system-they are highly sensitive to fluid shear gradients ${ }^{1,2}$ and proliferate significantly more under no-stress conditions ${ }^{3}$. Cells in endothelial tissue adhere tightly to their neighbours to prevent leakage, thus causing the cells to move highly collectively ${ }^{4-10}$. Because of this tight adhesion to neighbouring cells, mechanical forces are transmitted over large distances across the tissue ${ }^{11}$. Such a mechanical signal can serve as a guide for the cells as they most often prefer to migrate in the direction of least shear stress ${ }^{12}$ and seem to navigate towards empty spaces ${ }^{13}$. A mechanical signal transmitted across the tissue can cause the cells to deform ${ }^{14,15}$, move $\mathrm{e}^{21}$, divide ${ }^{17}$ and probably even differentiation can be mechanically controlled ${ }^{18,19}$. The living tissue is distinctly different from other materials as it consists of self-propelling cells that have a metabolism and can divide. Here we focus on how a cell division, which can be viewed as a local injection of energy, influences monolayer dynamics. The process of endothelial cell division is essential for correct embryo development ${ }^{20}$, angiogenesis and vessel repair ${ }^{21}$, as well as for the growth of metastasis from malignant tissue ${ }^{22}$.

\section{Results}

Dynamics and structure following cell division. To examine the effect of cell division on flows in the endothelial monolayer, we tracked the motility of cells surrounding a division site. The division site was centred in the analysed frame, and the frame was rotated such that the daughter cells initially (time 0 in Fig. 1a) move apart in a horizontal direction. The monolayer had an average cell density of $\sim 800$ cells $\mathrm{mm}^{-2}$, a density that does not limit cell division ${ }^{9}$, and each cell had on average 6 neighbours as shown by Voronoi analysis (Supplementary Fig. 1). We used particle image velocimetry (PIV) ${ }^{6,8-10,23}$ to track the collective motion of cells every $10 \mathrm{~min}$ between $80 \mathrm{~min}$ before and $80 \mathrm{~min}$ after the central cell's division. PIV analysis finds the maximum correlation between intensity patterns in two consecutive frames and returns the velocity field (shown as vectors in Fig. 1). We applied phase contrast microscopy, which facilitates the PIV analysis, particularly around cell nuclei. Time 0 is defined as the first image taken after cytokinesis where the cytoplasm of the mother cell is divided in two. Supplementary Figure 2 confirms that the PIV analysis correctly tracks the individual cells' trajectories within the confluent monolayer.

To increase the signal-to-noise ratio in the analysis of the longrange velocity fields, we aligned and averaged over 100 cell divisions. Figure $2 \mathrm{a}-\mathrm{c}$ shows the average nucleic positions of $n=100$ cell divisions (centred in the frame and rotated randomly, clockwise or anticlockwise, so that the dividing cells initially move in a horizontal direction) $20 \mathrm{~min}$ before (Fig. 2a), immediately a

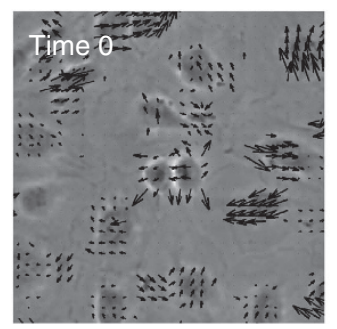

b

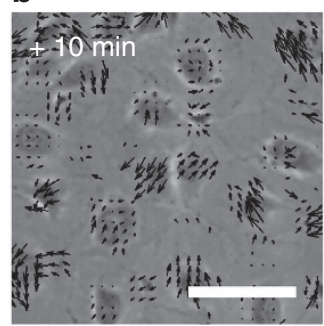

Figure 1 | Velocity field around a cell division in endothelial monolayer. (a) First image taken after cytokinesis (time 0 ), the dividing cell is in the center of the image. (b) Image taken $10 \mathrm{~min}$ after cytokinesis at the same location as $\mathbf{a}$. The vectors show the velocity field of the monolayer. The monolayer is confluent, all cells are connected with their nuclei (dark blobs) visually enhanced by phase contrast microscopy. Scale bar, $50 \mu \mathrm{m}$. after (Fig. 2b) and $20 \mathrm{~min}$ after (Fig. 2c) cytokinesis. During mitosis, the daughter nuclei move apart and in Fig. 1b a small counterclockwise rotation of the daughter cells with respect to the initial axis is visible, however, as detailed in Supplementary Fig. 3, the average rotation angle is $\left(4.5 \pm 16.3^{\circ}\right)$ which is not statistically significantly different from zero.

Divergence and vorticity fields. The divergence field measures the net flow across a boundary region and is calculated using equation (4) from Methods. Figure $2 \mathrm{~d}-\mathrm{f}$ shows the average divergence field of 100 aligned cells $10 \mathrm{~min}$ before (Fig. 2d), immediately after (Fig. 2e) and $30 \mathrm{~min}$ after (Fig. 2f) cytokinesis. The divergence analysis proves that the tissue contracts towards the division site before cytokinesis and expands from the site of the the daughter cells after cytokinesis. Additional time frames of the divergence field is shown in Supplementary Fig. 4 together with the corresponding nucleic positions.

The vorticity field (calculated by equation (5) in Methods) describes the curl of the velocity field and carries important information about tissue flows induced by cell division. At a certain time, $\sim 30 \mathrm{~min}$ after cell division, a distinct, long-range, well-ordered vortex pattern emerges as shown in cartesian (Fig. 3a) and polar coordinates (Fig. 3b), respectively. At the division site, there is no preferred sign of vorticity (as demonstrated in Supplementary Fig. 5). Adjacent to the division site two primary vortex pairs appear, with a clockwise (red) and a counterclockwise (blue) vortex flanking each daughter cell. These
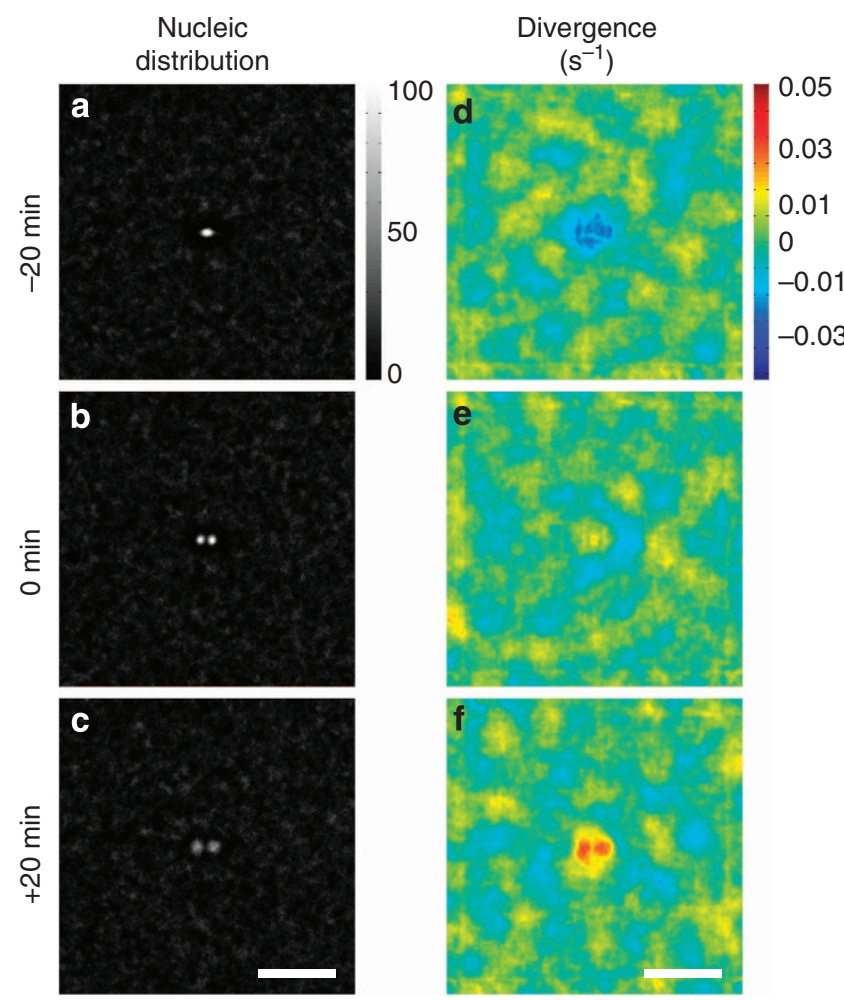

Figure 2 | Average nuclei positions and divergence of the velocity field around a cell division. In the center of each frame, a cell divides and the daughter cells initially move in a horizontal direction, each image is an average of 100 samples. (a-c) Images derived from phase contrast microscopy (the nuclei appear bright and the cytoplasm dark) displaying the average positions of nuclei 20 min before (a), immediately after (b) and 20 min after (c) cytokinesis initiation. (d-f) Divergence of the velocity field at corresponding times before cytokinesis initiation (d), immediately after (e) and longer after (f) cytokinesis initiation. Scale bars, $80 \mu \mathrm{m}$. 
a

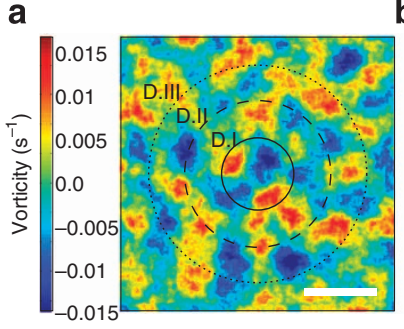

Experiment b

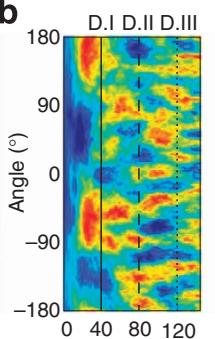

Radial distance $(\mu \mathrm{m})$ $c$

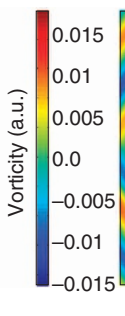

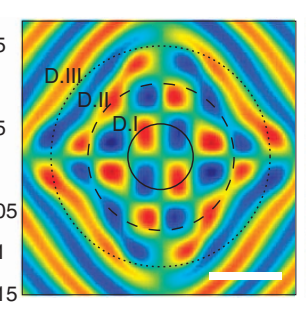

Simulation

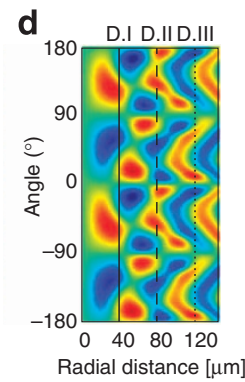

Figure 3 | Long-range well-ordered vorticity pattern induced by cell division. The vorticity field emerging 30 min after cell division shown in cartesian coordinates (a) and polar coordinates (b). Images are the results of averaging 100 samples around an aligned cell division site. The full (D.I), dotted (D.II) and dashed (D.III) lines denote distances one, two and three cell diameters away from the division site, respectively, corresponding to radii of 40,80 and $120 \mu \mathrm{m}$. (c,d) Vorticity field arising from a numerical simulation of the continuum model in cartesian coordinates (c) and in polar coordinates (d). Scale bars, $80 \mu \mathrm{m}$.
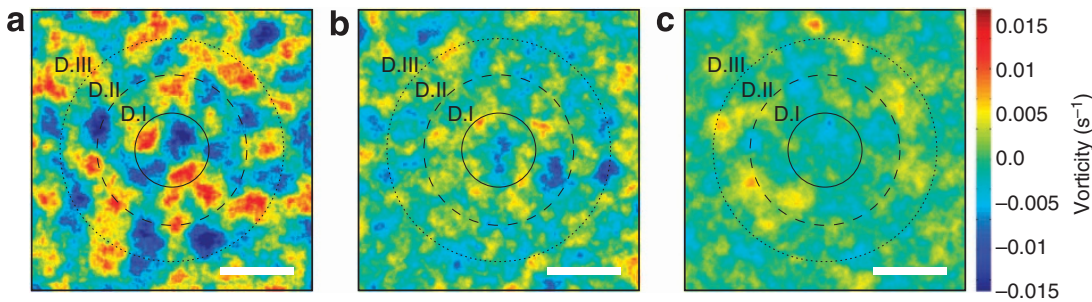

Figure 4 | Extinction of the vorticity pattern over time. The average vorticity field shown for (a) $30 \mathrm{~min}(n=100)$, (b) $2 \mathrm{~h}$ and $30 \mathrm{~min}(n=71)$, (c) $4 \mathrm{~h}$ and $30 \mathrm{~min}(n=71)$ after cell division. During time, the long-range ordering becomes more blurred and finally goes extinct at a time long enough after the cell division. Scale bars, $80 \mu \mathrm{m}$.

are located approximately one cell diameter away from the division site (D.I, full line in Fig. 3a,b). Well-ordered secondary and tertiary vortices are also induced by the cell division and appear farther from the division site: Approximately two cell diameters away (D.II, dashed lines in Fig. 3a,b), an ordered ring of eight vortex pairs is observed. Even at a distance of three cell diameters away from the division site (D.III, dotted lines in Fig. 3a,b) another ordered ring of vortices emerges, though at this distance from the central division center, the pattern is somewhat noisy due to the cell divisions taking place outside the framed region (no other cell divisions take place within the a distance of 6 cell diameters during the span of an experiment). A vortex is typically $\sim 40 \mu \mathrm{m}$, as is the cell diameter. Supplementary Video 1 shows, in parallel, the time evolution of a dividing cell, the average nuclei positions and the accompanying divergence and vorticity fields.

To verify that our alignment procedure did not influence the results, several controls were made (two are shown in Supplementary Fig. 6) with alternative rotations of the data sets, these controls yield results consistent with Fig. 3. Even if a smaller subset of the data was used, $n=30$, a similar pattern occurred (Supplementary Fig. 7). Tissue areas without dividing cells or tissues where cell division had been chemically prohibited were also examined. These controls (shown in Supplementary Fig. 8) had significantly smaller vorticity fields and no long-range, ordered vorticity patterns. Hence, the pattern observed around a cell division is significantly different from random noise in the tissue. Also, monitoring the evolution of the vorticity over longer timescales, up to $4-5 \mathrm{~h}$, as shown in Fig. 4 shows that the longrange ordered vorticity structures slowly lessen and disappear at times sufficiently long after cell division.

Continuum model. To understand the physical origin of these long-range, well-ordered vorticity patterns arising from a cell division site in a two-dimensional (2D) tissue we formulated a continuum model, which was inspired by recent theoretical work reproducing the dynamics of bacterial suspensions ${ }^{24}$. The dynamics of the tissue is assumed to satisfy a momentum balance equation:

$$
\partial_{t} \mathbf{v}=\frac{1}{\rho}(\nabla \cdot \sigma)-\left(\alpha+\beta|\mathbf{v}|^{2}\right) \mathbf{v},
$$

where $\rho$ is the mean density, $\mathbf{v}$ the local mean velocity of the tissue and $\sigma$ is the stress tensor. The latter two terms are parametrized by $\alpha$ and $\beta$ and can be thought of as the positive half of a double well potential in $|\mathbf{v}|$. Similar terms have also been employed to describe flocks and herds ${ }^{25}$. In this model, there is no inertial term, as the frictional forces totally dominate inertia for tissue movement. Stability requirements demand $\beta>0$, while $\alpha$ can have either sign. If $\alpha<0$, in addition to the isotropic equilibrium state $\mathbf{v}=0$, we get a non-trivial solution where cells move in an ordered state with a characteristic speed, $v_{c}=\sqrt{\frac{|\alpha|}{\beta}}$. Shortly after cell division, it is further assumed that the tissue moves as an incompressible fluid in which the projected area of each cell is conserved such that $\nabla \cdot \mathbf{v}=0$. The stress tensor, $\sigma$, in equation (1) is assumed to have the form ${ }^{24}$ :

$$
\begin{aligned}
\sigma_{i j}= & -p \delta_{i j}+\eta_{0}\left(\partial_{i} v_{j}+\partial_{j} v_{i}\right)-\eta_{2} \nabla^{2}\left(\partial_{i} v_{j}+\partial_{j} v_{i}\right) \\
& +S\left(v_{i} v_{j}-\frac{\delta_{i j}}{D}|\mathbf{v}|^{2}\right),
\end{aligned}
$$

where $p$ is the pressure. The linear momentum diffusion is parameterized by $\eta_{0}$ and a higher-order dissipative term $\eta_{2}$, a nematic term, similar to the Q-tensor for nematic crystals ${ }^{26}$, including a fitting parameter, $S$, and the spatial dimension of the setup, $D=2$, which describes the active stress contribution ${ }^{24}$. Comparing experimental data with the simulation, we find the fitted value $S / \rho \sim 2.1 \pm 0.2$. In a tissue, the cells are self-propelling and continuously inject energy to move against viscous forces. 

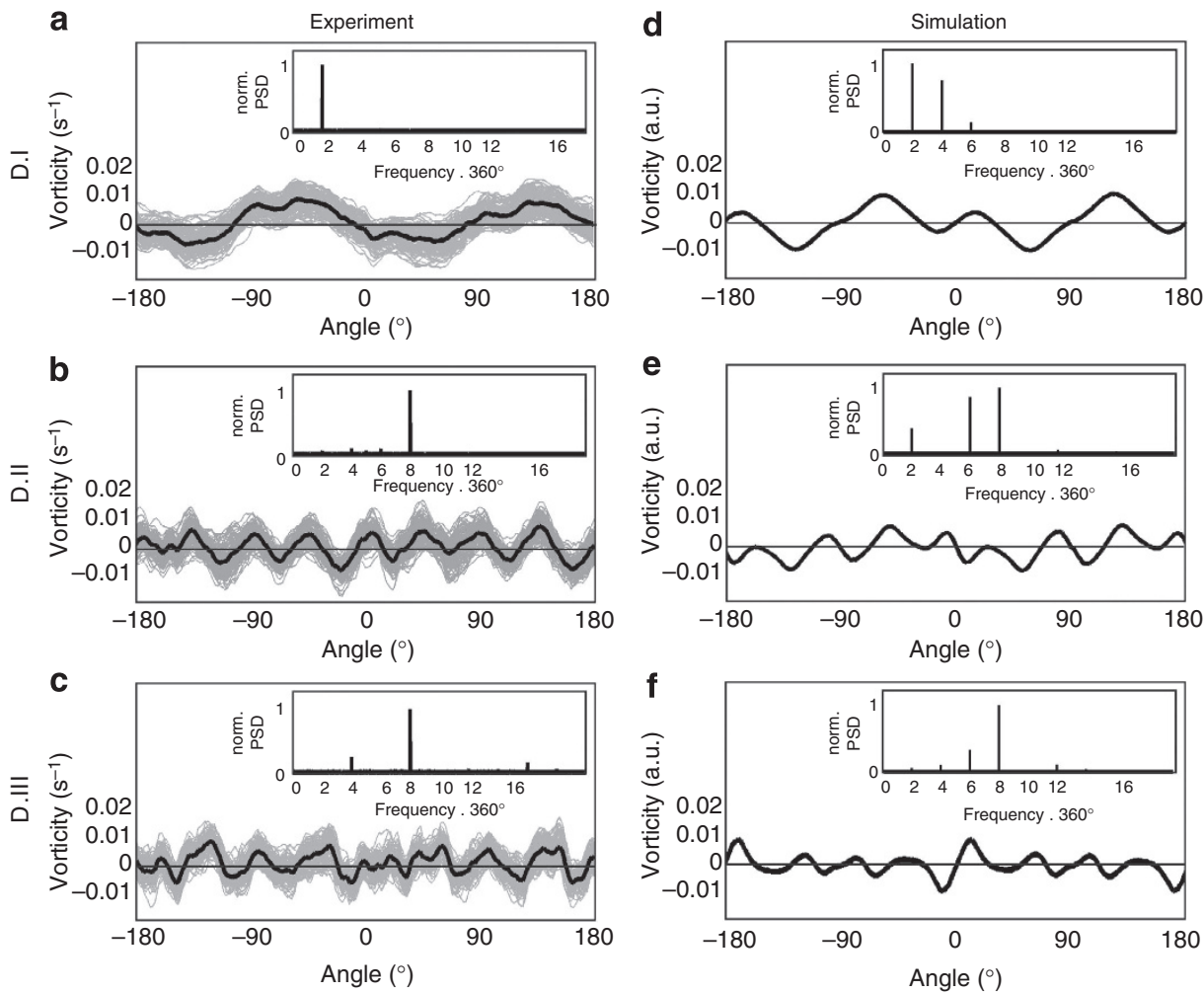

Figure 5 | Quantification of primary, secondary and tertiary induced vortices. Vorticity as function of angle at radial distances of 1 (a), 2 (b) and 3 (c) cell diameters away from the cell division site $30 \mathrm{~min}$ after cell division. One hundred and twenty-nine different averages, each of 100 cells, are shown in light grey, their average is shown by a full black line. (d-f) the vorticity stemming from numerical simulation of the continuum model at the same distance from the division site as a-c, respectively. The insets show the corresponding power spectral densities.

In accordance with classical literature ${ }^{27-29}$ (and with the more recent ref. 24), we model this behaviour by setting $\eta_{0}<0$. This negative viscosity, which is here introduced into our model, arises from the classical method of describing systems in which the fluctuation spectrum has a low non-zero extremal value and in which the viscosity can be renormalized through an expansion around the extremal point ${ }^{27-29}$. This expansion gives rise to an unstable second-order term and a higher-order stabilizing term (where the latter can be interpreted as a viscosity). In our experiments, a length scale of the order of a cell diameter sets the extremal point in the fluctuation spectrum, and we switch on the higher-order term $\eta_{2}>0$ to ensure stability of the system. This causes the two uniform solutions to become unstable and form a dynamic state resembling tissue dynamics. Inserting the stress tensor into equation (1) yields the following model:

$$
\begin{aligned}
\partial_{t} \mathbf{v}= & -\frac{1}{\rho} \nabla p+\frac{S}{\rho}(\mathbf{v} \cdot \nabla) \mathbf{v}-\frac{S}{\rho D} \nabla|\mathbf{v}|^{2} \\
& +v_{0} \nabla^{2} \mathbf{v}-v_{2} \nabla^{4} \mathbf{v}-\left(\alpha+\beta|\mathbf{v}|^{2}\right) \mathbf{v}
\end{aligned}
$$

where $v_{0}=\eta_{0} / \rho$ and $v_{2}=\eta_{2} / \rho$.

The symmetry is broken during cell division when the two daughter cells migrate in opposite directions. To model the cell division, we locally add a stokeslet dipole, $f(t)(\boldsymbol{\delta}(\mathbf{x}+\mathbf{a})$ $-\boldsymbol{\delta}(\mathbf{x}-\mathbf{a}))$, on the right-hand side of equation (3). The amplitude $f(t)$ has the form of a square pulse in time and gives rise to two oppositely oriented forces (see details in Methods). The stokeslet dipole is aligned with the cell division axis and gives rise to a symmetry breaking. This pertubation locally and temporarily violates incompressibility; however, the system quickly relaxes and incompressibility is recovered.

One difference between our model and previous variants of the above equations $24,25,30,31$ is that our model considers the dynamics of a dense packing of cells, whereas previous works have focused on dilute suspensions of self-propelled particles. Our model is valid in a limit where the divergence of the elastic stress is balanced by the frictional forces between the cells and the substrate. Also, the velocities of interest here are approximately eight orders of magnitude slower than the elastic wave in the cytoplasm $^{32}$. More details on the model, a stability analysis and explanation of the implementation of the numerical simulation of equation (3) are given in the Methods section.

The proposed model nicely captures the physics of the tissue dynamics and quantitatively reproduces the long-range vorticity pattern after cell division as shown in Fig. 3c. Also, the radial vorticity plot (Fig. $3 \mathrm{~d}$ ) is reproduced. The parameters entered into the model are the time of interest $(30 \mathrm{~min}$ to compare with Fig. 3a,b) and the size of the analysed image $(300 \times 300 \mu \mathrm{m})$. A best fit of our model to the data returns an average speed of $1.4 \mu \mathrm{mmin}^{-1}$, which corresponds well to the experimentally observed average speed of $0.9 \mu \mathrm{m} \mathrm{min}^{-1}$. In addition, the velocity field, both the overall central patterns of the vector field and the absolute peak values, is well reproduced by the simulation (see Supplementary Fig. 9). This set of model parameters also returns the same divergence values as experimentally observed after cell division (Fig. 2).

Fourier analysis. To further quantify the induced long-range ordered vorticity pattern, we performed a Fourier analysis of the vorticity in bands at approximately one, two and three cell diameters away from the division site, these are shown in Fig. $5 \mathrm{a}-\mathrm{c}$. The grey lines in Fig. 5 show the vorticity as function of angle for 129 alternative rotations, the full black line shows their average. The simulated vorticity versus angle is shown in Fig. 5d-f. Both the experimental and the simulated vorticities 
have a periodic pattern and to determine the periodicity the corresponding power spectral densities were found (insets of Fig. 5). These quantify the number vortex pairs as two primary, eight secondary and eight tertiary vortex couples located one, two and three cell diameters away from the division site as visually apparent in Fig. 3a,b. Again, the numerical model reproduces the experimental data. Even if a smaller data set, $n=30$, is used, the same features are very clear in the power spectral analysis (Supplementary Fig. 10). Hence, the model captures the essential physics of tissue dynamics and this, combined with our experimental results, proves that the endothelial tissue displays hydrodynamic properties as a connected biomaterial rather than as a collection of individual cells.

\section{Discussion}

The motility of cells in a confluent cellular monolayer is known to correlate over distances of $\sim 200-350 \mu \mathrm{m}$ (refs 6,7), and correlated swirls exist over distances up to $\sim 400 \mu \mathrm{m}$ (ref. 6). In light of these distances, it is not surprising that the motion of new-born daughter cells stirs the endothelial monolayer and orders the tissue at distances of up to $140 \mu \mathrm{m}$ away from the division site. As the daughter cells move apart in the endothelial monolayer, they exert a drag-force on the adhering neighbouring cells, thus creating two primary vortex couples. Such primary vortex couples have been reported for two cylinders moving apart in a continuous viscoelastic sheet ${ }^{33}$. However, the additional emergence of secondary and tertiary vortices in a predominantly viscous sheet has, to our knowledge, never been observed in a biological system before, but only in hydrodynamical turbulent systems characterized by high Reynolds numbers ${ }^{34}$. Using a characteristic speed of $1 \mu \mathrm{m} \mathrm{min}-1$, a typical cell size of $40 \mu \mathrm{m}$ (or a system size of $300 \mu \mathrm{m}$ ) and a cytoplasmatic viscosity of 1417 poise (for human umbilical vein endothelial cells in the absence of vascular endothelial growth factor $)^{35}$, we estimate a Reynolds number characterizing endothelial monolayer dynamics to be $\sim 10^{-9}$; hence, tissue dynamics cannot be viewed as classical turbulence. However, as the tissue is not a Newtonian fluid, the dynamics is not well characterized by this Reynolds number.

Our continuum model that treats cell division as a local pressure increase, captures the tissue dynamics, both the velocity and vorticity fields. Tissue flows determine the orientation of the cells, and thereby of their division axes ${ }^{17}$. The orientation of the cell division axis could be decisive for whether a vessel lengthens or becomes thicker, hence, for the ability of an organism, for example, to counteract the clotting of a vessel, and this is a property that should be mimicked in artificial vessels. In literature, long-range tissue communications are attributed to chemical signalling ${ }^{36}$; however, our results show that the physical properties of tissue alone can explain long-range ordering and cellular communication. As stem cell differentiation can be mechanically regulated $^{37}$ and the mechanisms governing morphogenesis are related to vortex formation ${ }^{20}$, it may be that the hydrodynamical ordering following cell division also influences differentiation and morphogenesis.

\section{Methods \\ Cell culture. Human umbilical vein endothelial cells (Invitrogen) were cultured in T25 flasks (Nunclon) with Endothelial Cell Basal Medium (Cell Applications). To create confluent monolayers, $\sim 100,000$ cells were seeded in Collagen IV-coated 30 -mm circular dishes and cultured for 3 days at $37^{\circ} \mathrm{C}$ and $5 \% \mathrm{CO}_{2}$ with a media change every $24 \mathrm{~h}$}

Experiments. All experiments were conducted 3 days after seeding the cells. Phase contrast images $\left(2,500 \times 3,000 \mu^{2}\right)$ were taken of the monolayer every $10 \mathrm{~min}$ for $8 \mathrm{~h}$. All dividing cells in the monolayer were located in the phase contrast images using a custom written MatLab programme that recognized the rounded cell before mitosis. Only isolated dividing cells, that is, cells that had no other dividing cell within a $240-\mu \mathrm{m}$ radius for $20 \mathrm{~min}$ before to $30 \mathrm{~min}$ after, were used for this analysis. Over 1,000 dividing cells were identified and followed for $80 \mathrm{~min}$ before and after division, 100 of which could be considered 'isolated' using these restrictions.

Image analysis. Image sequences of dividing cells were cropped from the larger phase contrast images. These images were aligned by centring the dividing cell in a $300 \times 300 \mu \mathrm{m}^{2}$ frame and rotating the frame so that the two daughter cells move away from the site of division along the horizontal axis immediately after mitosis (as shown in Fig. 1a). The rotation could in principle be done in two ways, either by rotating an angle $\alpha$ clockwise or an angle 180- $\alpha$ counterclockwise. We chose at random between the two and performed several controls where rotations were done differently (examples are shown in Supplementary Fig. 6).

Particle image velocimetry. We used PIV to calculate the vector field describing the displacement of the cells' nuclei between images taken $10 \mathrm{~min}$ apart. We used the PIVlab software package (www.mathworks.com/matlabcentral/fileexchange/ 27659-pivlab-time-resolved-particle-image-velocimetry-piv-tool) for MATLAB (The MathWorks, Natick, MA) and used an interrogation area of $15.4 \times 15.4 \mu \mathrm{m}^{2}$ corresponding to $24 \times 24$ pixels $^{2}$. The displacement and velocity vectors were calculated for each pixel.

Divergence and vorticity. From the velocity vectors, we calculated the divergence and vorticity of the flow field. The divergence and vorticity were calculated for each pixel using the characteristic length scale of the system, the radius of the average cell area. If the cell density of a sample is 800 cells $\mathrm{mm}^{-2}$, then the cells have an average area of $A=1,250 \mu \mathrm{m}^{2}$, and the center of two neighbouring cells will be $\sim 40 \mu \mathrm{m}$ apart on average. The divergence, $d$, has SI units of $\left(\mathrm{s}^{-1}\right)$ and measures the net flow (in units of $\left(\mathrm{m}^{2} \mathrm{~s}-1\right)$ ), of the vector field across the smooth boundary of a small region, $A$, divided by its area in units of $\left(\mathrm{m}^{2}\right)$. Hence, $d$ was computed as:

$$
d=\frac{\partial}{\partial x} v_{x}+\frac{\partial}{\partial y} v_{y}=\sum_{\mathbf{r} \in O} \frac{v_{x}(\mathbf{r}) \mathbf{r}_{\mathbf{x}}+v_{\mathbf{y}}(\mathbf{r}) \mathbf{r}_{\mathbf{y}}}{A},
$$

in which $O$ is the circumference of the spherical area, $A, \mathbf{r}=\left(\mathbf{r}_{x}, \mathbf{r}_{y}\right)$ is the vector from the center of $A$ to a point on $O$ and $\overrightarrow{\mathbf{v}}$ is the velocity vector on that point on $O$.

The strength of the vorticity, $\omega=\left(s^{-1}\right)$, was computed as the amount of

circulation, $\Gamma=\left(\mathrm{m}^{2} \mathrm{~s}^{-1}\right)$, along the boundary of a small region, divided by the area of the small region:

$$
\omega=\frac{\Gamma}{A}=\frac{\oint \mathbf{v}(\mathbf{r}) \cdot d \mathbf{r}}{A}=\sum_{\mathbf{r} \in O} \frac{v_{x}(\mathbf{r}) \mathbf{r}_{\mathbf{y}}-v_{\mathbf{y}}(\mathbf{r}) \mathbf{r}_{\mathbf{x}}}{A} .
$$

More details on the continuum model. From ref. 32, we deducted the velocity of an elastic wave in the cytoplasm to be $1 \mathrm{~m} \mathrm{~s}^{-1}$. The length and timescales of interest for the current work are $10 \mu \mathrm{m}$ over $10 \mathrm{~min}$, hence, eight orders of magnitude slower than the elastic wave. Therefore, our model essentially followed from a balance between the divergence of the elastic stress and frictional forces, and not inertia. In the model, we assume the frictional forces to be proportional to the negative cell velocity and the stress to be proportional to the gradients in the displacement. Applying a time derivative to this balance gives an equation where the cell acceleration is proportional to the divergence of gradients in the velocity field. This essentially gives equation (1) where we in addition include contributions from the self-propelling force of the individual cells.

By taking the divergence of the vector field on both sides of equation (1), we achieve a modified version of the conventional Poisson equation for the pressure field encountered for incompressible Newtonian fluid flow,

$$
\nabla^{2} p=\sum_{i j} S\left(\partial_{i} v_{j}\right)\left(\partial_{j} v_{i}\right)-\beta v_{j}\left(v_{i} \partial_{i}\right) v_{j}-\frac{S}{D} \nabla^{2}|\mathbf{v}|^{2} .
$$

Experimentally, the dividing cell locally breaks the symmetry and chooses an axis along which the two daughter cells migrate in opposite directions. We model the cell division process as a stokeslet dipole, that is, by a local force perturbation along the $x$ axis such that

$$
\nabla \cdot \sigma \rightarrow \nabla \cdot \sigma+f(t)(\boldsymbol{\delta}(x+a, y)-\boldsymbol{\delta}(x-a, y)),
$$

where we have introduced an amplitude $f(t)$ of the local force and two $\delta$ functions. The amplitude of the force perturbation has the shape of a square pulse in time $f(t)=f_{0}$ for $0 \leq t \leq t_{\text {division }}$ otherwise $f(t)=0$, where we set $t_{\text {division }}=1 \mathrm{~min}$. $f_{0}$ is fitted such that the resulting amplitude of the velocity perturbation matches the experimentally measured amplitude. The scale $a$ was in our simulation set to $7 \mu \mathrm{m}$ in real units. In the simulation, the dipole is aligned with the axis of migration of the two new daughter cells.

By inserting the stress tensor, equation (2), in equation (1) we arrive at equation (3), which describes the tissue dynamics. Coupling equation (3) to the incompressibility equation, $\nabla \cdot \mathbf{v}=0$, gives the continuum model used in the $2 \mathrm{D}$ simulation. Note that we need both the second- and fourth-order derivatives to describe the cell motion. Those two terms introduce a length scale comparable to 
the cell size, below which the dynamics is stable. That is, on the scale of individual cells, the dynamics is fairly coherent, whereas on larger scales the dynamics is controlled by unstable wavenumbers generating an inhomogeneous state of the velocity field. In the $2 \mathrm{D}$ case, this can be made further apparent by taking the curl of equation (3) linearized around the $\mathbf{v}=0$ solution

$$
\partial_{t} \omega=-v_{2}\left(\nabla^{2}-\frac{v_{0}}{2 v_{2}}\right)^{2} \omega-\left(\alpha-\frac{v_{0}^{2}}{4 v_{2}}\right) \omega .
$$

For $v_{0}<0$, this term will generate vorticity structures with an approximate wavelength of $2 \pi \sqrt{\frac{2 v_{2}}{\left|v_{0}\right|}} \sim 1.5$ cell diameters, which will describe a tendency for nearby cells to rotate in opposite directions. As can be seen from the stability analysis below, the long-range ordered state does not form when the second-order term is stabilizing the dynamics, that is, when $v_{0}>0$.

Stability analysis. To examine further the dynamics of the model, we performed a linear stability analysis of equation (3) around a velocity field $\mathbf{v}_{0}$, which is independent of space and time $\mathrm{e}^{24,38}$. Therefore, all derivatives in equation (3) vanish and the pressure computed from equation (6) is constant. Equation (3) then simplifies to

$$
\left(\alpha+\beta\left|\mathbf{v}_{0}\right|^{2}\right) \mathbf{v}_{0}=0,
$$

which has two solutions when $\beta>0$ and $\alpha<0,\left|\mathbf{v}_{0}\right|=0$ and $\left|\mathbf{v}_{0}\right|=\sqrt{\frac{|\alpha|}{\beta}}$. We now perform a stability analysis of the these two solutions by perturbing both the velocity and the pressure fields by a small variation, $\mathbf{v}=\mathbf{v}_{0}+\delta \mathbf{v}$ and $p=p_{0}+\delta p$. To simplify the analysis, the coordinate system is rotated such that $\mathbf{v}_{\mathbf{0}}=\left(v_{0}, 0\right)$ and $\delta \mathbf{v}=\left(\epsilon_{\|}, \epsilon_{\perp}\right)$, with $\epsilon_{\|}, \epsilon_{\perp}$ being the parallel and perpendicular components of the perturbation with respect to the velocity vector. Fourier transforming equation (3), we find to linear order in $\delta \mathbf{v}$

$$
\begin{aligned}
\partial_{t} \delta \hat{\mathbf{v}}= & -\left(v_{0} k^{2}+v_{2} k^{4}-i S k_{x} v_{0}\right) \delta \hat{\mathbf{v}}-i\left(\delta \hat{p}+\frac{2 S v_{0}}{D} \epsilon_{\|}\right) \mathbf{k} \\
& -\left(\alpha+\beta\left(v_{0}^{2}+2 v_{0} \epsilon_{\|}\right)\right)\left(\mathbf{v}_{0}+\delta \hat{\mathbf{v}}\right),
\end{aligned}
$$

where the ' $\wedge$ ' denotes transformed quantities and $k^{2}=k_{x}^{2}+k_{y}^{2}$. For $v_{0}=0$, we find from the incompressibility condition, $\nabla \cdot \mathbf{v}=0$, that the growth of the perturbation is on the form $|\delta \hat{\mathbf{v}}| \propto \exp (\lambda t)$, where $\lambda=-\left(\alpha+v_{0} k^{2}+v_{2} k^{4}\right)$. For $v_{0}<0$ and $v_{2}>0$, the uniform solution will be unstable to perturbations with wavenumbers in a narrow interval. For $v_{0}=\sqrt{\frac{|\alpha|}{\beta}}$ the last term in equation (10) can be simplified into

$$
\partial_{t} \delta \hat{\mathbf{v}}=-\left(v_{0} k^{2}+v_{2} k^{4}-i S k_{x} v_{0}\right) \delta \hat{\mathbf{v}}-i\left(\delta \hat{p}+\frac{2 S v_{0}}{D} \epsilon_{\|}\right) \mathbf{k}+\left(\begin{array}{cc}
2 \alpha & 0 \\
0 & 0
\end{array}\right) \delta \hat{\mathbf{v}} .
$$

Again using the incompressibility condition, we find that

$$
\partial_{t} \delta \hat{\mathbf{v}}=-\mathbf{M}\left(\left(v_{0} k^{2}+v_{2} k^{4}-i S k_{x} v_{0}\right) \mathbf{I}-\left(\begin{array}{cc}
2 \alpha & 0 \\
0 & 0
\end{array}\right)\right) \delta \hat{\mathbf{v}},
$$

where $\mathbf{I}$ is the identity matrix and

$$
\mathbf{M}=\left(\begin{array}{cc}
1-\frac{k_{x}^{2}}{k^{2}} & -\frac{k_{x} k_{y}}{k^{2}} \\
-\frac{k_{k} k_{y}}{k^{2}} & 1-\frac{k_{y}^{2}}{k^{2}}
\end{array}\right) .
$$

The eigenvalues for the non-zero solution are $\lambda=0$ and $\lambda=-\left(v_{0} k^{2}+v_{2} k^{4}-i S k_{x} v_{0}\right)$ $+2 \alpha_{\frac{k}{k^{2}}}$. Once again for $v_{0}<0$ and $v_{2}>0$, we find a region of unstable wavenumbers making both fixed points simultaneously unstable. In Supplementary Fig. 11, we show the results of the stability analysis where the region of the unstable wavenumbers can be observed for the two fixed points, respectively. We further observe that for $\alpha<0$ and $\eta_{0}<0$, both fixed points will be unstable when perturbed by long wavelengths implying that equation (3) describes an inhomogeneous velocity field.

Model parameter estimation. The five model parameters with a physical dimension are given by

$$
\begin{aligned}
& {[L]=\text { length, } \quad[\alpha]=\frac{1}{\text { time }}, \quad[\beta]=\frac{\text { time }^{\text {length }^{2}}, \quad\left[v_{0}\right]=\frac{\text { length }^{2}}{\text { time }} \text { and }}{}} \\
& {\left[v_{2}\right]=\frac{\text { length }^{4}}{\text { time }} .}
\end{aligned}
$$

In general, $\alpha$ and $\beta$ describe the properties of the isotropic states, while $v_{0}$ and $v_{2}$ describe the emergence and evolution of the vorticity patterns. Combining the dimensional quantities, we find that the velocity of the ordered state is given by $v_{c}=\sqrt{\frac{|\alpha|}{\beta}}$, which roughly corresponds to the crawling velocity of the cells.

The length scale $L$ is set to size of the experimental system. The four remaining parameters have been fitted by minimizing the distance between the vorticity field of the model and the experiments in a circle with a radius of approximately three cell diameters and centred on a cell division event. To convert the dimensionless model parameters to real parameters with units, we use a timescale and the length scale $L$. In the experiment, the vorticity pattern develops fully over the course of $\sim t=30 \mathrm{~min}$, which corresponds to $t=11.4 \cdot t_{\mathrm{s}}$ in simulation time. From that we estimate the time conversion factor from the simulation time to be $t_{\mathrm{s}}=2.63 \mathrm{~min}$. The physical parameters and their corresponding physical values and SI units are provided in Supplementary Table 1 . The full simulation spans a $4 \pi \times 4 \pi$ square. To ensure that the boundary effects from the simulation's periodic boundaries are negligible, only the central square region of the simulation is used. The length conversion factor is found to be $l_{\mathrm{s}} \approx 47.7 \mu \mathrm{m}$. Using the conversion factors, $t_{\mathrm{s}}$ and $l_{\mathrm{s}}$, and the values from Supplementary Table 1, we find the characteristic cell speed to be $\mathrm{v}_{c} \approx 1.4 \mu \mathrm{m} \mathrm{min}^{-1}$ in good agreement with the experimental value $\left(0.9 \mu \mathrm{m} \mathrm{min}^{-1}\right)$.

Numerical simulation. The model was simulated in a 2D box with periodic boundary conditions using a pseudospectral method. After Fourier transforming equation (1), the resulting equations were solved numerically by an exponential time integration scheme ${ }^{39}$. The non-linear terms were evaluated in real space and then transformed back to Fourier space by repeated use of the Fast Fourier Transform and its inverse. To suppress aliasing errors, the 3/2-rule has been implemented ${ }^{40}$. The stability of the simulations were tested for a wide range of parameters and on grid sizes ranging from $128 \times 128$ to $512 \times 512$ with timesteps of the order $\Delta t \sim 10^{-4}$. To ensure that the flow field remained incompressible, a pressure correction term was implemented effectively driving the divergence of the velocity field towards zero ${ }^{40}$. The simulation was initialized with a divergence-free flow field, which was allowed to relax. Thereafter, the perturbation in the pressure was inserted and the flow field relaxed once more, while the vorticity field was extracted.

\section{References}

1. DePaola, N., Gimbrone, M. A., Davies, P. F. \& Dewey, C. F. Vascular endothelium responds to fluid shear stress gradients. Arterioscler. Thromb. Vasc. Biol. 12, 1254-1257 (1992).

2. Davies, P. F., Remuzzi, A., Gordon, E. J., Dewey, C. F. \& Gimbrone, M. A. Turbulent fluid shear stress induces vascular endothelial cell turn over in vitro. Proc. Natl Acad. Sci. USA 83, 2114-2117 (1986).

3. Akimoto, S., Mitsumata, M., Sasaguri, T. \& Yoshida, Y. Laminar shear stress inhibits vascular endothelial cell proliferation by inducing cyclin-dependent kinase inhibitor p21. Circ. Res. 86, 185-190 (2000).

4. Szabó, A. et al. Collective cell motion in endothelial monolayers. Phys. Biol. 7, 046007 (2010)

5. Vitorino, P. \& Meyer, T. Modular control of endothelial sheet migration. Genes Dev. 22, 3268-3281 (2008)

6. Angelini, T. E., Hannezo, E., Trepat, X., Fredberg, J. J. \& Weitz, D. A. Cell migration driven by cooperative substrate deformation patterns. Phys. Rev. Lett. 104, 168104 (2010).

7. Vitorino, P., Hammer, M., Kim, J. \& Meyer, T. A steering model of endothelial sheet migration recapitulates monolayer integrity and directed collective migration. Mol. Cell. Biol. 31, 342-350 (2011).

8. Angelini, T. E. et al. Glass-like dynamics of collective cell migration. Proc. Natl Acad. Sci. USA 108, 4714-4719 (2011).

9. Poujade, M. et al. Collective migration of an epithelial monolayer in response to a model wound. Proc. Natl Acad. Sci. USA 104, 15988-15993 (2007).

10. Reffay, M. et al. Orientation and polarity in collectively migrating cell structures: statics and dynamics. Biophys. J. 100, 2566-2575 (2011).

11. Trepat, X. et al. Physical forces during collective cell migration. Nat. Phys. 5, 426-430 (2009).

12. Tambe, D. T. et al. Collective cell guidance by cooperative intercellular forces. Nat. Mater. 10, 469-475 (2011).

13. Kim, H. J. et al. Propulsion and navigation within the advancing monolayer sheet. Nat. Mater. 12, 856-863 (2013).

14. Kasza, K. E. et al. The cell as a material. Curr. Opin. Cell Biol. 19, 101-107 (2007).

15. Tseng, P., Judy, J. W. \& Carlo, D. D. Magnetic nanoparticle-mediated massively parallel mechanical modulation of single-cell behavior. Nat. Methods $\mathbf{9}$, 1113-1119 (2012).

16. Serra-Picamal, X. et al. Mechanical waves during tissue expansion. Nat. Phys. 8, 628-634 (2012).

17. Fink, J. et al. External forces control mitotic spindle positioning. Nat. Cell Biol. 13, 771-778 (2011).

18. Lanniel, M. et al. Substrate induced differentiation of human mesenchymal stem cells on hydrogels with modified chemistry and controlled modulus. Soft Matter 7, 6501-6514 (2011).

19. Gonzalez-Cruz, R., Fonseca, V. \& Darling, E. Cellular mechanical properties reflect the differentiation potential of adipose-dericed mesenchymal stem cells Proc. Natl Acad. Sci. USA 109, E1523-E1529 (2012).

20. Chuai, M. et al. Cell movement during chick primitive streak formation. Dev. Biol. 296, 137-149 (2006).

21. Gerhardt, H. \& Betsholtz, C. in Mechanisms of Angiogenesis. (eds Clauss, M. \& Breier, G.) Chapter 8 (Birkhauser, 2005). 
22. Folkman, J. Tumor angiogenesis: therapeutic implications. N. Engl. J. Med. 285, 1182-1186 (1971)

23. Perryn, E. D., Czirók, A. \& Little, C. D. Vascular sprout formation entails tissue deformations and VE-cadherin-dependent cell-autonomous motility. Dev. Biol. 313, 545-555 (2008).

24. Wensink, H. et al. Meso-scale turbulence in living fluids. Proc. Natl Acad. Sci. USA 109, 14308-14313 (2012).

25. Toner, J. \& Tu, Y. Flocks, herds, and schools: a quantitative theory of flocking. Phys. Rev. E 58, 4828-4858 (1998).

26. de Gennes, P. G. \& Prost, J. The Physics of Liquid Crystals (Clarendon Press, 1993)

27. Brazovskji, J. Phase transition of an isotropic system to a nonuniform state. Sov. Phys. JETP 41, 85-89 (1975).

28. Swift, J. \& Hohenberg, P. C. Hydrodynamic fluctuations at the convective instability. Phys. Rev. A 15, 319-328 (1977).

29. Kuramoto, Y. \& Tsuzuki, T. Persistent propagation of concentration waves in dissipative media far from thermal equilibrium. Progr. Theor. Phys. 55, 356-369 (1976).

30. Simha, R. A. \& Ramaswamy, S. Hydrodynamic fluctuations and instabilities in ordered suspensions of self-propelled particles. Phys. Rev. Lett. 89, 058101 (2002).

31. Pedley, T. J. Instability of uniform micro-organism suspensions revisited. J. Fluid Mech. 647, 335-359 (2010)

32. Zeng, D. et al. Young's modulus of elasticity of Schlemms canal endothelial cells. Biomech. Model Mechanobiol. 9, 19-33 (2010).

33. Thomases, B. \& Shelley, M. Emergence of singular structures in Oldroyd-B fluids. Phys. Fluids 19, 103103 (2007).

34. Brede, M., Eckelmann, H. \& Rockwell, D. On secondary vortices in the cylinder wake. Phys. Fluids 8, 2117-2124 (1996).

35. Wirtz, D. Particle-tracking microrheology of living cells: principles and applications. Annu. Rev. Biophys. 38, 301-326 (2009).

36. Zeng, G. et al. Orientation of endothelial cell division is regulated by vegf signaling during blood vessel formation. Blood 109, 1345-1352 (2007).

37. Sun, Y. et al. Mechanics regulates fate decisions of human embryonic stem cells. PLoS ONE 7, e37178 (2012).

38. Dunkel, J., Heidenreich, S., Bär, M. \& Goldstein, R. Minimal continuum theories of structure formation in dense active fluids. New J. Phys. 15, 045016 (2013).
39. Pope, D. A. An exponential method of numerical integration of ordinary differential equations. Commun. ACM 6, 491-493 (1963).

40. Henshaw, W. D. \& Petersson, N. A. Numerical Simulation of incompressible Flows (World Scientific, 2003).

\section{Acknowledgements}

We would like to thank S. Loft and H. Klingberg for the human umbilical vein endothelial cell line, J.S. Juul, H. Klingberg and M. Shelley for insightful discussions and J. Yeomans for sharing details about the meso-scale turbulence model with us. We acknowledge financial support from the University of Copenhagen Excellence programme and from the Danish Research Councils.

\section{Author contributions}

N.S.R. and L.B.O. designed the research. N.S.R. carried out the experiments and conducted the analysis. J.M. and M.H.J. proposed the model and J.M.T. carried out the simulation. N.S.R., J.M., J.M.T. and L.B.O. wrote the manuscript. M.H.J, J.M. and L.B.O. oversaw the project.

\section{Additional information}

Supplementary Information accompanies this paper at http://www.nature.com/ naturecommunications

Competing financial interests: The authors declare no competing financial interests.

Reprints and permission information is available online at http://npg.nature.com/ reprintsandpermissions/

How to cite this article: Rossen, N. S. et al. Long-range ordered vorticity patterns in living tissue induced by cell division. Nat. Commun. 5:5720 doi: 10.1038/ncomms6720 (2014).

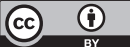

This work is licensed under a Creative Commons Attribution 4.0 International License. The images or other third party material in this article are included in the article's Creative Commons license, unless indicated otherwise in the credit line; if the material is not included under the Creative Commons license, users will need to obtain permission from the license holder to reproduce the material. To view a copy of this license, visit http://creativecommons.org/licenses/by/4.0/ 